\title{
Coincident onset of multiple sclerosis and Herpes simplex virus 1 encephalitis: a case report
}

\author{
M. C. Buscarinu ${ }^{1 *}$, A. Fornasiero ${ }^{1}$, S. Romano ${ }^{1}$, M. Ferraldeschi ${ }^{3}$, R. Renié ${ }^{1}$, G. Trasimeni ${ }^{4}$, M. Salvetti ${ }^{1,2}$ and G. Ristori ${ }^{1}$
}

\begin{abstract}
Background: Along with vitamin D, smoking, body mass index and others, Epstein Barr virus, other herpesviruses and human endogenous retroviruses represent plausible environmental risk factors for multiple sclerosis. However, it is difficult to obtain direct proof of their involvement in the etiology of this condition.

Case presentation: In order to contribute further evidence of the importance of these viruses, and speculate about disease-relevant interactions between these agents and a predisposed genetic background of the host, we describe the temporal association between multiple sclerosis onset and Herpes simplex 1-encephalitis in a female patient.

Conclusions: This case illustrates a possible relationship between HSV-1 encephalitis and multiple sclerosis. Bearing in mind that association does not imply causation, some speculations about the etiology and pathophysiology of the two diseases can be made. The hypothesis of a genetic background predisposing to HSV-1 encephalitis and to immune-mediated demyelination is supported by the coincidence of the two conditions in this patient, along with data from animal models and genetic studies.
\end{abstract}

Keywords: Multiple sclerosis, Environmental factors, HSV-1 encephalitis

\section{Background}

Heritable and nonheritable factors, and their interactions, may be responsible for the autoimmune response that leads to multiple sclerosis (MS) [1].

Genome-wide association studies are improving our understanding of the heritable basis of the disease, most probably involving subtle defects of the regulation of transcription [2]. Though these studies are indeed advancing our knowledge, the nonheritable or "environmental" component of the risk shows a slower progress. Among the environmental risk factors, viruses are intensively investigated as possible culprits [3]. Human endogenous retroviruses such as MSRV have attracted interest because of their capacity to hide by integrating in the human DNA and then trigger immunological mechanisms: a scenario that is compatible with the long standing difficulties in detecting any infectious agent in

\footnotetext{
* Correspondence: mariachiara.buscarinu@uniroma1.it

${ }^{1}$ Centre for Experimental Neurological Therapies, Department of

Neuroscience, Mental Health and Sensory Organs, Faculty of Medicine and

Psychology, Sapienza University of Rome, Rome, Italy

Full list of author information is available at the end of the article
}

the MS central nervous system. On the other hand, herpesviruses are sound candidates as well, because of neurotropism and, in the case of Epsein Barr virus, extraordinary ability to modulate the human immune response.

In order to contribute further evidence of the importance of these viruses, and speculate about diseaserelevant interactions between these agents and a predisposed genetic background of the host, we describe the temporal association between MS onset and Herpes simplex 1 (HSV)-encephalitis in a female patient.

\section{Case Presentation}

A 32 year-old woman presented acute dysesthesia in the right face. Except for this symptom, her neurological examination was unremarkable and neither the patient nor her relatives could recall any previous neurological episode. Her neurologist requested an MRI of the brain and spinal cord that showed the simultaneous presence of enhancing and nonenhancing white matter abnormalities in MS-typical regions of the brain, compatible with the diagnosis of a first episode of the disease (Fig. 1). 


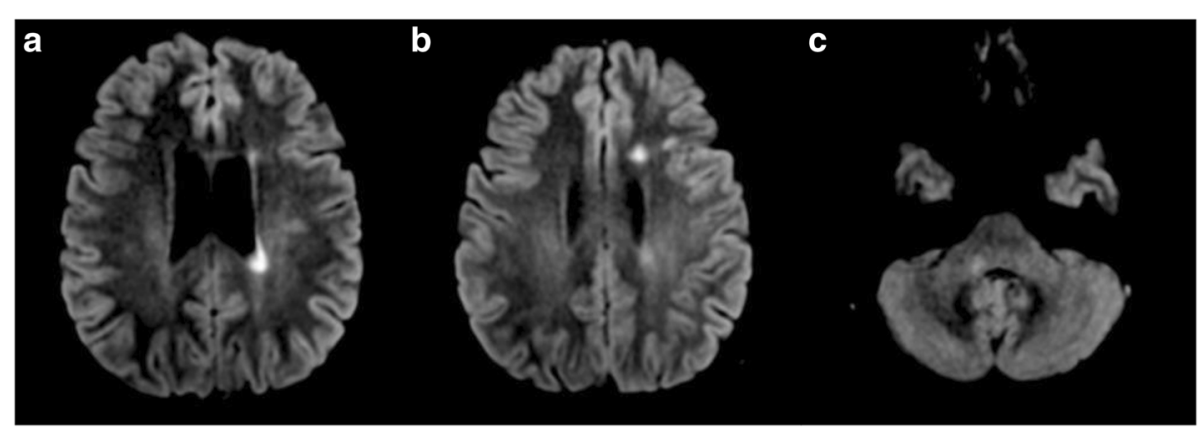

Fig. 1 a Axial FLAIR MRI that highlights hyperintense left periventricular areas; b left periventricular and frontal subcortical; c right cerebellar peduncle

She was then treated with methylprednisolone $1 \mathrm{~g}$ intravenously for five days with a clear improvement of the symptom. Ten days after the completion of the corticosteroid course, she presented fever, severe headache and confusion. A brain CT scan was obtained, highlighting a hypodense area that involved right temporal pole, insula and hippocampus. She was then hospitalized and her cerebrospinal fluid (CSF) tested positive for HSV-1 DNA. All other CSF tests were negative including: Cytomegalovirus, Varicella Zoster, Epstein Barr virus, John Cunningham virus, enteroviruses, Toxoplasma, Cryptococcus, Mycobacterium tuberculosis and Treponema, oligoclonal bands. With the exception of a positive test for recent HSV-1 infection, all other serologies were negative for recent infections including: Coxiella, Coxsackie, Chlamydia pneumoniae, Borrelia burgdoferi, Mycoplasma pneumoniae, echovirus and adenovirus. Peripheral blood screening tests for autoimmune diseases were negative.

Intravenous acyclovir was started but she rapidly worsened (GCS: 4; E1, V1, M2) requiring orotracheal intubation, tracheostomy, mechanical ventilation and antiepileptic and antiedema therapy. Brain MRI obtained 3 and 6 days after the CT scan showed the involvement of the right hemisphere with diffuse swelling and contralateral shift of the septum pellucidum (Fig. 2).

After 22 days, HSV1 DNA in the CSF was negative while a third MRI, contemporary to the CSF sampling, showed a remarkable increase in the number and volume of lesions in MS-typical areas (some of them enhancing) and necrotic-malacic evolution of the herpetic lesion (Fig. 3)

In view of the apparent flare of the "autoimmune" condition, the patient was started on intravenous immunoglobulins (IVIG) at $0.4 \mathrm{gr} / \mathrm{kg} /$ daily for 5 days and Methilprendisolone $40 \mathrm{mg} /$ daily for 15 days. She also continued therapy with Valacyclovir $3 \mathrm{~g} /$ daily.

The MRI carried out as a control at the end of intravenous steroid therapy confirmed the necrotic-malacic evolution of the right temporal lesion, with a reduction of the brainstem lesions and of the corpus callosum, and with evidence of a C5 spinal cord area, compatible with an inflammatory lesion. The enhancement of lesions in MS-typical regions was not evident at this point (Fig. 4).

She was transferred to a rehabilitation facility (GCS: 7) and, after ten months, discharged home with a remarkable improvement: left hemiparesis and left sensory syndrome but able to walk with assistance, short term memory loss, fluctuating hyperactive state with mild agitation. After additional six months of follow-up, she has now slightly improved the strength of her left limbs. The number and volume of areas in MS-typical regions is reduced though still increased with respect to the first MRI and no new areas have appeared at brain and spinal cord MRI follow-up (Fig. 5).

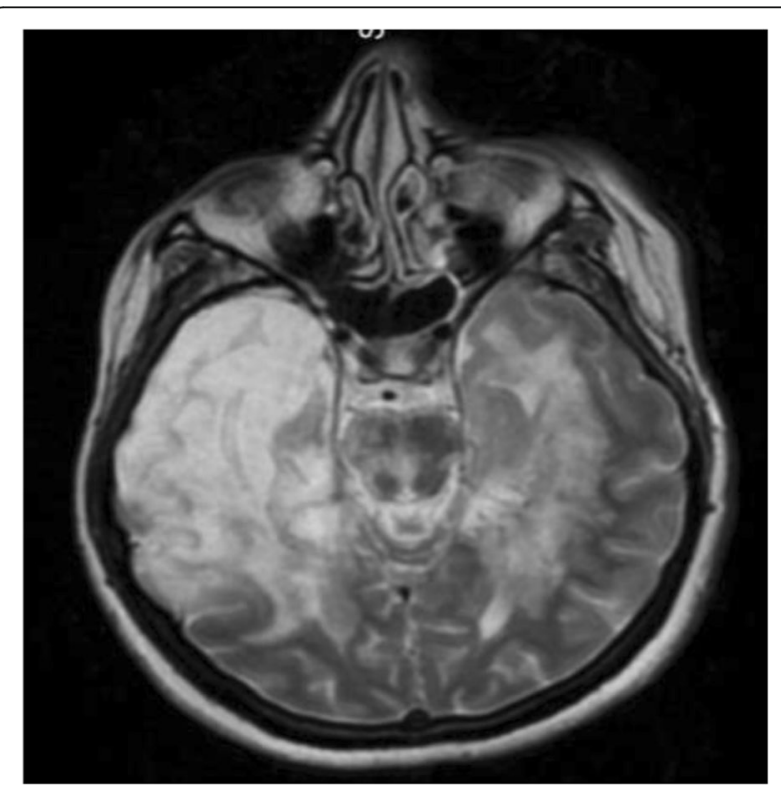

Fig. 2 Axial T2 image showing hyperintensity of the right temporal lobe and right brainstem with less obvious contralateral alterations 


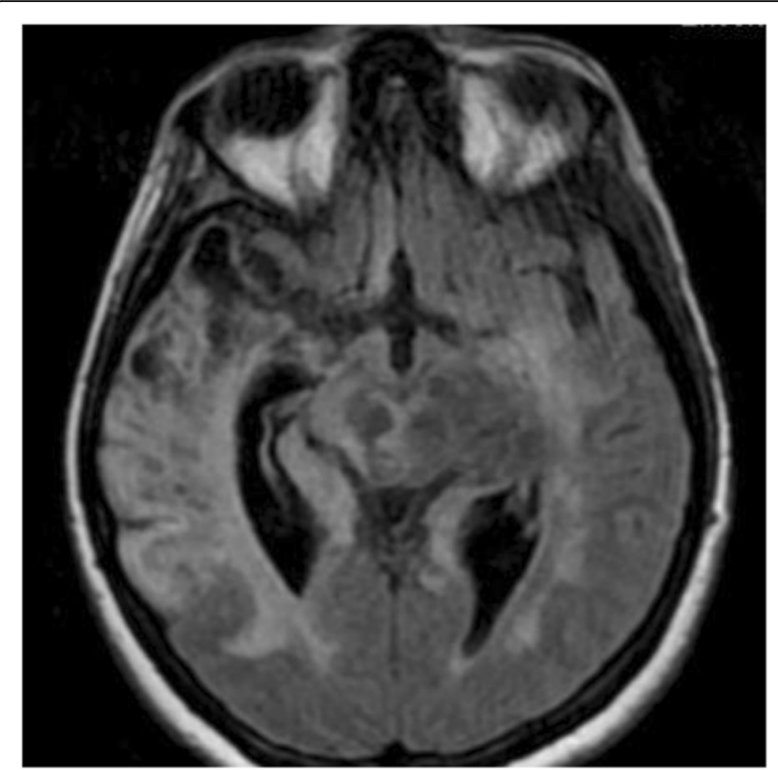

Fig. 3 Axial FLAIR showing the temporal encephalitis outcome

\section{Discussion and conclusions}

This case illustrates a possible relationship between HSV-1 encephalitis and MS.

Bearing in mind that association does not imply causation, some speculations about the etiology and pathophysiology of the two diseases can be made.

To our knowledge, while the onset of MS after hemithorax zoster, with intrathecal reactivation of HSV-1 and HHV-6 has been reported [4] this is the first description

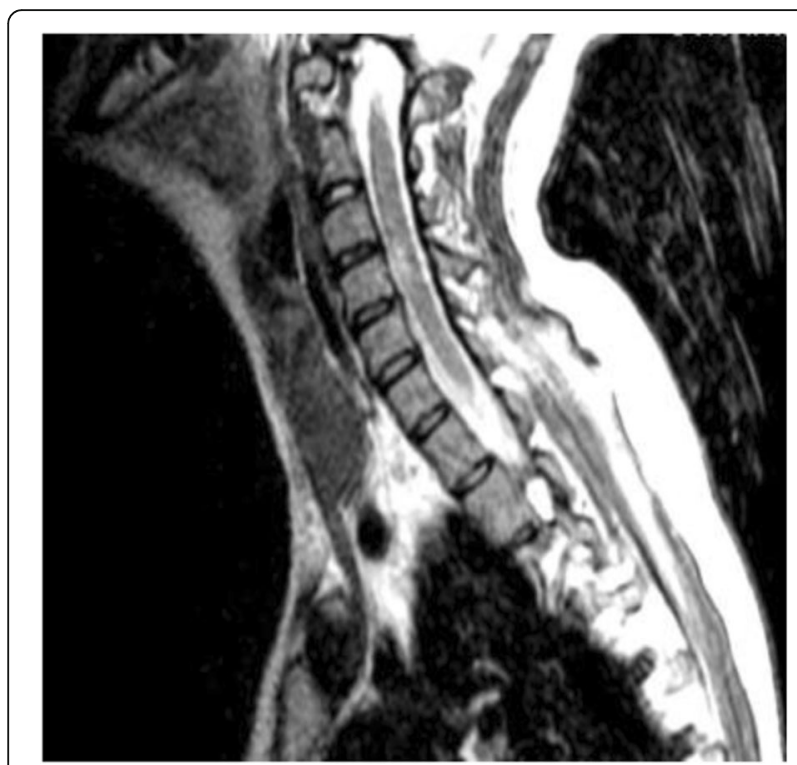

Fig. 4 Cervical spinal cord sagittal image showing demyelinating hyperintense areas at $\mathrm{C} 4$ of a coincident onset of MS and HSV-1 encephalitis. Other reports have provided laboratory evidence of the intrathecal presence of HSV-1 at disease onset. This supports the possibility that, in an individual with a peculiar genetic predisposition, this presence may turn into overt HSV-1 encephalitis [5, 6]. However, other viruses such as the John Cunningham (JC) virus have been described as replicating in the cerebrospinal fluid during the first symptoms of MS [7] though a coincident onset of MS and progressive multifocal encaphalomyelitis have never been described to our knowledge.

Most interestingly, the HSV-1 encephalitis develops in individuals with inborn errors of immune response genes that impair central nervous system-intrinsic interferon alpha/beta production [8]. The therapeutic response of persons with MS to interferon beta suggests that a portion of the pathophysiology of the two diseases may be shared. This possibility may be supported also by the sharing of a predisposing gene, TRAF3, between the two conditions (though this speculation has to be taken with caution because the effects of the observed variants may not be the same) $[8,9]$.

The role of a genetic predisposition emerges also from studies in inbred mouse strains with variable degrees of resistance to EAE/HSV [10]. In these models, the lip inoculation of the virus causes either no disease, or isolated lesions, or a diffuse picture of demyelination, depending on the genetic background of the mice. Interestingly, the multifocal lesions in the susceptible mice were first observed when the virus could no longer be isolated from the CNS of the animals. This is in line with the diffuse worsening of the bona-fide demyelinating lesions observed in the patient, when the viral DNA became undetectable in the CSF. Both observations are in harmony with an immune-mediated process, triggered by the virus, in hosts with a susceptible genetic background. Other sequences of events cannot be excluded: the autoimmune damage might have been present before the HSV-1 infection with the latter being exacerbated by the corticosteroids course. Nonetheless, the effects of corticosteroids in these patients are debated, as clinical trials are ongoing to assess their therapeutic effects in overt HSV-1 encephalitis. Also the role played by the diffuse alteration of the blood brain barrier (somehow resembling an acute disseminated encephalomyelitis process, secondary to the HSV-1 infection) remains unclear in the context of the whole process. A longitudinal follow-up with, for example, IEF-overlay technique with radiolabeled HSV glycoprotein B (gB), for studying qualitative aspects of the HSV humoral immune response $[11,12]$, would have been of use but was not performed. More in general, as common pathophysiologies emerge between MS and host immune response to viruses, tests for the detection of antiviral antibodies may acquire more importance in the near future. 


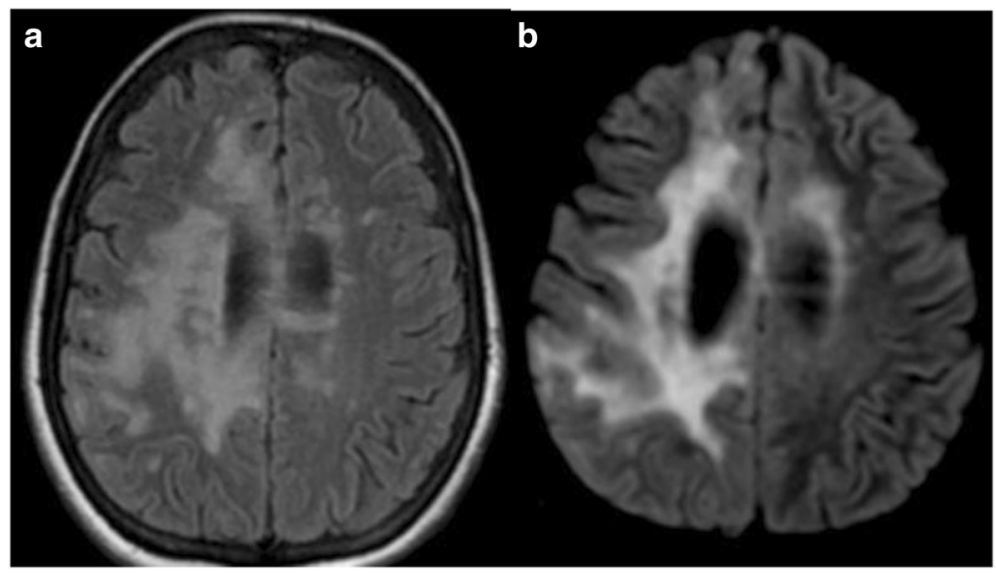

Fig. 5 Comparison of MRls obtained at 4 months (a) and at 18 months after onset (b) showing a reduction of the areas in MS-typical regions though the lesion load remains higher compared with the first MRI (Fig. 1)

All in all, the hypothesis of a genetic background predisposing to HSV-1 encephalitis and to immunemediated demyelination is supported by the coincidence of the two conditions in this patient, along with data from animal models and genetic studies.

\section{Acknowledgements}

The authors would like to thank the patient for consent for publication.

\section{Funding}

No funding was received.

\section{Availability of data and material}

Not applicable.

\section{Authors' contributions}

$M C B, M S$ and GR: contributed equally for writing and critical reading of the manuscript; GT provided interpretation of MRI imaging; AF, SR, MF, RR: provided critical reading of the manuscript. All authors read and approved the final manuscript

\section{Competing interests}

The authors declare that they have no competing interests.

\section{Consent for publication}

Informed consent was obtained from the patient for publication of this case report and any accompanying images.

\section{Ethics approval and consent to participate}

Not applicable.

\section{Publisher's Note}

Springer Nature remains neutral with regard to jurisdictional claims in published maps and institutional affiliations.

\section{Author details}

${ }^{1}$ Centre for Experimental Neurological Therapies, Department of Neuroscience, Mental Health and Sensory Organs, Faculty of Medicine and Psychology, Sapienza University of Rome, Rome, Italy. ${ }^{2}$ IRCCS Istituto Neurologico Mediterraneo (INM) Neuromed, Pozzilli, Italy. ${ }^{3}$ Department of Neurology and Psychiatry, Sapienza University of Rome, Rome, Italy. ${ }^{4}$ Neuroradiology Unit, Department of Neuroscience, Mental Health and Sensory Organs, Faculty of Medicine and Psychology, Sapienza University of Rome, Rome, Italy.
Received: 2 February 2017 Accepted: 30 March 2017 Published online: 28 April 2017

\section{References}

1. Bordi I, Ricigliano VA, Umeton R, Ristori G, Grassi F, Crisanti A, Sutera A, Salvetti M. Noise in multiple sclerosis: unwanted and necessary. Ann Clin TransI Neurol. 2014;1 (7):502-11. doi:10.1002/acn3.72. Epub 2014 Jun 19.

2. Farh KK, Marson A, Zhu J, Kleinewietfeld M, Housley WJ, Beik S, Shoresh N, Whitton H, Ryan RJ, Shishkin AA, Hatan M, Carrasco-Alfonso MJ, Mayer D, Luckey CJ, Patsopoulos NA, De Jager PL, Kuchroo VK, Epstein CB, Daly MJ, Hafler DA, Bernstein BE. Genetic and epigenetic fine mapping of causal autoimmune disease variants. Nature. 2015;518(7539):337-43. doi:10.1038/ nature13835. Epub 2014 Oct 29.

3. Salvetti M, Giovannoni G, Aloisi F. Epstein-Barr virus and multiple sclerosis. Curr Opin Neurol. 2009;22(3):201-6. doi:10.1097/WCO.0b013e32832b4c8d.

4. Ferrò MT, Franciotta D, Prelle $A$, Bestetti $A$, Cinque P. Active intrathecal herpes simplex virus type 1 (HSV-1) and human herpesvirus-6 (HHV-6) infection at onset of multiple sclerosis. J Neurovirol. 2012;18:437-40. doi:10.1007/s13365-012-0110-5.

5. Franciotta D, Bestetti A, Sala S, Perucca P, Jarius S, Price RW, Di Stefano AL, Cinque P. Broad screening for human herpesviridae DNA in multiple sclerosis cerebrospinal fluid and serum. Acta Neurol Belg. 2009;109:277-82.

6. Bergström $T$, Andersen $\mathrm{O}$, Vahlne A. Isolation of herpes simplex virus type 1 during first attack of multiple sclerosis. Ann Neurol. 1989;26:283-5. doi:10.1002/ana.410260218

7. Ribeiro T, Fleury MJ, Granieri E, et al. Investigation of the prevalence of antibodies against neurotropic polyomaviruses BK, JC and SV40 in sera from patients affected by multiple sclerosis. Neurol Sci. 2010;31(4):517-21. doi:10.1007/s10072-010-0353-y.

8. Alcaïs A, Abel L, Casanova JL. Human genetics of infectious diseases: between proof of principle and paradigm. J Clin Invest. 2009:119:9.

9. Zhang SY, Casanova JL. Inborn errors underlying herpes simplex encephalitis: From TLR3 to IRF3. INSIGHTS |. J Exp Med. 2015;24.

10. Kastrukoff LF, Lau AS, Kim SU. Multifocal CNS demyelination following peripheral inoculation with herpes simplex virus type 1. Ann Neurol. 1987;22(1):52-9.

11. Grimaldi LME, Roos RP, Manservigi R, Spear PG, Lakeman FD, Whitley RJ. An Isoelectric Focusing Study in Herpes Simplex Virus Encephalitis. Ann Neurol. 1988:24:2.

12. Martino GV, Grimaldi LME, Franciotta DM, Piccolo G, Brustia R, Minali L, GV M d'E. Isoelectric focusing herpes simplex virus-gB overlay study in brainstem encephalitis. Acta Neurol Scand. 1990;82(6):364-7. 